Z Rheumatol 2010 -69:195-195

DOI 10.1007/s00393-010-0620-6

๑) Springer-Verlag 2010

\author{
U. Müller-Ladner \\ Lehrstuhl für Innere Medizin mit Schwerpunkt Rheumatologie der \\ Justus-Liebig-Universität Gießen, Abteilung für Rheumatologie und \\ Klinische Immunologie, Kerckhoff-Klinik GmbH, Bad Nauheim
}

\title{
Bewährtes und Perspektiven
}

Sehr geehrte Kolleginnen und Kollegen, liebe Leserinnen und Leser,

wenn man gefragt wird, ob Interesse bestünde, für ein renommiertes Journal die Aufgabe des Schriftleiters zu übernehmen, kann die Antwort eigentlich nur positiv ausfallen. Da die Zeitschrift für Rheumatologie gleichzeitig das Organ unserer Gesellschaft ist, hat mich dieses Angebot und das hiermit verbundene Vertrauen natürlich besonders gefreut. Leichtgefallen ist mir die Annahme dieses Angebots auch deshalb, da Herr Prof. Gross und sein Team in Zusammenarbeit mit den hervorragenden Mitarbeitern des Springer-Verlags, der DGRh, der DGKJ, der ARO und der Rheumaliga sowie vielen externen Kollegen einen Standard aufgebaut haben, der eigentlich nicht zu verbessern ist. Zudem haben wir den Luxus, ein Editorial Board - zu dem

\section{Das Redaktionsteam \\ "Zeitschrift für Rheumatologie"}

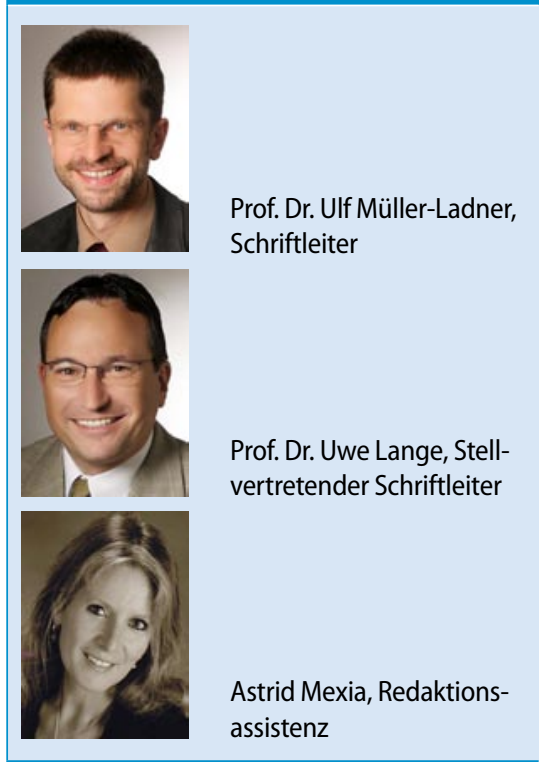

selbstverständlich auch Herr Prof. Gross, Herr PD Dr. Moosig und Herr Prof. Dr. Hellmich weiterhin angehören werden zur zukünftigen Gestaltung an der Hand zu haben, das den häufig gebrauchten Titel „key experts“, bzw. „key opinion leaders" mehr als verdient.

Sie werden sich nun sicher fragen, welche Neuerungen durch oder mit dem neuen Editorial Board eingeführt werden. Hier waren bzw. sind zunächst Sie gefragt, uns - in welcher Form auch immer - Rückmeldung zu geben, in welchen Bereichen Sie Bedarf für Verbesserungen sehen, denn die Zeitschrift soll ja primär Ihre Bedürfnisse nach Fortbildung und Kommunikation erfüllen. Parallel hierzu wird die elektronische Artikeleinreichung und -Review eingeführt, so dass die Neuigkeiten aus dem Gebiet der Kinderund Erwachsenenrheumatologie/klinischen Immunologie und den Nachbargebieten Sie zukünftig noch schneller erreichen werden.

Die bewährten Rubriken unter der Leitung der Rubrikherausgeber bleiben natürlich erhalten, werden aber durch zwei weitere besondere Rubriken, die direkt dem Editorial Board unterstellt sind, ergänzt: Zum einen durch das „Hot Topic", in dem ein/e Experte/in zu einem aktuellen Thema einen prägnanten Kommentar unter „Peer-review-Bedingungen“ erstellen wird, der zum Nachdenken und zur weiteren Diskussion anregen soll. Des Weiteren wird es eine Rubrik „Labortechniken" geben, in der möglichst anschaulich und mit leicht verständlichen Illustrationen eine für die Praxis wichtige Technik, z. B. Immunfluoreszenz, erklärt wird, um den nicht mehr aktiv an der Laborbank Stehenden die Entstehung wichtiger Laborwerte nahe zu bringen.
Da die Rubrikeditoren bereits eine zuverlässige Größe für jede Ausgabe sind, betrifft der für Sie sichtbare Wechsel neben dem Schriftleiter vor allem das zentrale Team in der Schriftleitung, und ich freue mich besonders, dass Herr Prof. Uwe Lange das Angebot des stellvertretenden Schriftleiters angenommen hat. Dies bedeutet auch, dass wir mit kurzen Wegen in der Redaktion das gesamte Feld der klinischen und experimentellen Rheumatologie, der klinischen Immunologie, Osteologie, physikalischen Therapie und Balneologie in der täglichen Kommunikation mit Ihnen abdecken können. Besonders wichtig ist auch, dass mit Frau Astrid Mexia eine Redaktionsassistentin gefunden werden konnte, die nicht nur in der internationalen Kommunikation erfahren ist, sondern sich auch durch ihr hervorragendes Organisationstalent hier vor Ort bei mehreren Veranstaltungen ausgezeichnet hat.

Kurz gesagt, ich freue mich auf die Zusammenarbeit mit Ihnen und bin überzeugt, dass die Zeitschrift für Rheumatologie auch in der Zukunft mehr als nur Ihre monatliche Lektüre sein wird.

Für das Redaktionsteam

Ihr

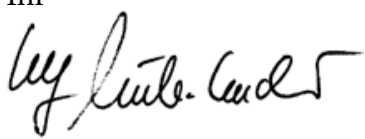

Ulf Müller-Ladner

\section{Korrespondenzadresse}

Prof. Dr. U. Müller-Ladner

Lehrstuhl für Innere Medizin mit Schwerpunkt Rheumatologie der Justus-Liebig-Universität Gießen, Abteilung für Rheumatologie und Klinische Immunologie, Kerckhoff-Klinik GmbH Benekestr. 2-8, 61231 Bad Nauheim u.mueller-ladner@kerckhoff-klinik.de 